\title{
The current state of cancer family history collection tools in primary care: a systematic review
}

\author{
Nadeem Qureshi, MBBS, DM ${ }^{1}$, June C. Carroll, $M^{2}$, Brenda Wilson, FFPH ${ }^{3}$, \\ Pasqualina Santaguida, PhD ${ }^{4,5}$, Judith Allanson, FRCP ${ }^{6}$, Melissa Brouwers, PhD ${ }^{4,7}$, \\ and Parminder Raina, $P h D^{4,5}$
}

\begin{abstract}
Systematic collection of family history is a prerequisite for identifying genetic risk. This study reviewed tools applicable to the primary care assessment of family history of breast, colorectal, ovarian, and prostate cancer. MEDLINE, EMBASE, CINAHL, and Cochrane Central were searched for publications. All primary study designs were included. Characteristics of the studies, the family history collection tools, and the setting were evaluated. Of 40 eligible studies, 18 relevant family history tools were identified, with 11 developed for use in primary care. Most collected information on more than one cancer and on affected relatives used self-administered questionnaires and paperbased formats. Eleven tools had been evaluated relative to current practice, demonstrating 46-78\% improvement in data recording over family history recording in patient charts and $75-100 \%$ agreement with structured genetic interviews. Few tools have been developed specifically for primary care settings. The few that have been evaluated performed well. The very limited evidence, which depends in part on extrapolation from studies in settings other than primary care, suggests that systematic tools may add significant family health information compared with current primary care practice. The effect of their use on health outcomes has not been evaluated. Genet Med 2009:11(7): 495-506.
\end{abstract}

Key Words: primary care, cancer genetics, family history, questionnaire, interview

A positive family history is a risk factor for many chronic diseases, reflecting "the consequences of genetic susceptibilities, shared environment, and common behaviors."1 The systematic collection and assessment of family history information is a potentially valuable tool in preventive medicine and is crucial in the identification of genetic risk. ${ }^{2}$ In some situations,

\footnotetext{
From the ${ }^{1}$ Division of Primary Care, University of Nottingham, Nottingham, United Kingdom; ${ }^{2}$ Granovsky Gluskin Family Medicine Centre, Mount Sinai Hospital, University of Toronto, Toronto, Canada; ${ }^{3}$ Department of Epidemiology and Community Medicine, University of Ottawa, Ottawa, Canada; ${ }^{4}$ Department of Clinical Epidemiology \& Biostatistics, Faculty of Health Sciences, McMaster University, Hamilton, Canada; ${ }^{5}$ McMaster University Evidence-Based Practice Centre, Faculty of Health Sciences, McMaster University, Hamilton, Canada; ${ }^{6}$ Children's Hospital of Eastern Ontario, Ottawa, Canada; and ${ }^{7}$ Program in Evidence-Based Care, Cancer Care Ontario, McMaster University, Hamilton, Canada.

Nadeem Qureshi, MBBS, DM, University of Nottingham, Graduate Medical School, Derby City General Hospital, United Kingdom DE22 3DT. E-mail: nadeem.qureshi@nottingham.ac.uk.
}

The opinions expressed herein do not necessarily reflect the opinions of the Agency for Healthcare Research and Quality.

The authors declare no conflict of interest.

Submitted for publication November 13, 2008.

Accepted for publication March 18, 2009.

Published online ahead of print June 10, 2009.

DOI: $10.1097 /$ GIM.0b013e3181a7e8e0 family history information alone can form the basis for offering patients appropriately tailored preventive interventions. ${ }^{1,3}$ In addition, the clinical predictive value of even the most accurate DNA test depends on penetrance of the gene mutation and requires family history information for test interpretation. ${ }^{4}$ Thus, family history information is potentially useful both as a clinical tool in its own right and also as an important adjunct to DNA-based testing.

Cancers are a group of relatively common conditions in which, for at least some, family history seems to be an important risk factor. This report focuses on four types of cancer: breast, colorectal, ovarian, and prostate. These are some of the most common cancers where the role of family history is recognized as a risk factor. $^{5-9}$ In some families, these cancers form part of recognized hereditary syndromes. ${ }^{9}$ In some cases, ethnic ancestry is also associated with risk of cancer-associated genetic mutation, such as breast cancer in the Ashkenazi Jewish community. ${ }^{10-13}$

Family history taking can be conducted as part of a diseasespecific approach, which aims to identify risk of selected single gene disorders (e.g., hereditary breast or colon cancer) for the purpose of offering appropriate specialist referral and management. ${ }^{14,15}$ Alternatively, it can be directed more broadly toward identifying possible multifactorial risk of common disorders such as cancer and diabetes. ${ }^{16,17}$ The extent of cancer family history collection has to be adequate to enable primary care providers (PCPs) to make appropriate clinical and prevention decisions, but it is not clear whether this necessarily requires the same approach as that used by a genetic specialist. ${ }^{3}$

This review explored how well the different systematic family history collection tools improve nonsystematic approaches to family history collection by PCPs. Both the format of available family history collection tools and comparison of these tools against current "optimal" and "standard" clinical practice were evaluated.

\section{MATERIALS AND METHODS}

\section{Literature search}

Search strategies were developed and undertaken for the time period 1990 to July 2007 in several electronic databases including: (1) Cochrane Central, (2) MEDLINE®, (3) EMBASE, and (4) CINAHL $\AA$. In addition to the electronic databases, the bibliographies of retrieved articles were reviewed, as well as key internet websites, for additional relevant articles. Keywords included prostate or breast or colorectal or ovarian and neoplasm or cancer, medical history taking, family health, pedigree, and genetic assessment or evaluation.

\section{Study selection}

Studies were eligible if they were published in English and included any of the four cancer populations of interest (breast, colorectal, ovarian, and prostate). Primary studies (both qualitative and quantitative) were eligible, but narrative and systematic reviews, editorials, letters (with no primary data), com- 
ments, opinions, abstracts and unpublished studies were excluded. Studies that focused on capturing, collecting, collating information on disease history, or illness in other family members by any method (self-report or by a professional) were eligible. We defined a family history tool as a systematic approach used to capture and document family history, appropriate for the clinical setting, with the potential to lead to decision making by a clinician.

Studies within primary care "clinical" settings or undertaken by the PCPs were eligible. Similarly, studies that identified tools that systematically collected family history in other settings but could be applicable to the primary care setting were also eligible. Full details of eligibility criteria are presented in the full Agency for Healthcare Research and Quality report. ${ }^{18}$ Also, relevant web-based tools were identified by exploring the gray literature and signposting by members of the project team and the external Technical Expert Panel. Applicability of tools in nonprimary care settings and web-based tools were assessed by two independent reviewers against five criteria: length of tool, ease of completion, need for specialist knowledge to complete tool, whether it was designed to capture data on at least all first degree relatives (1DRs), and clarity of layout (including appropriate structure and logical sequence). Articles were excluded if (a) there was a primary focus on genealogy (nonmedical family history), (b) they included mention of family history in some form but did not describe a tool or measure for use in clinical settings, (c) they were statistical or epidemiological studies designed to explore associations or test hypotheses relating to family history and cancer risk but did not describe a tool or measure, and (d) they reported data on patient psychosocial outcomes relating to cancer risk or family history taking, but did not describe a tool or measure. Trained reviewers independently evaluated citations at all levels of screening and any disagreements were resolved by consensus. Standardized forms within Systematic Review Software (SRS 4.0, TrialStat Corporation, Ottawa, Ontario, Canada) and reviewer guides were used to screen abstracts and studies and to extract data.

\section{Data abstraction and quality assessment}

Data extraction and quality assessment for all studies meeting the eligibility criteria were completed by two independent reviewers and standardized forms were used. The specific quality assessment scale used was dependent on the study design and the research question. Full details of quality assessment are given in the full report of the systematic review. ${ }^{18}$

\section{Data extraction}

A qualitative descriptive approach was used to summarize study characteristics and outcomes. Multiple publications on the same study cohort were grouped together and treated as a single study with the most current data reported for presentation of summary results. Two aspects of the studies using or evaluating family history tools were explored: descriptive data on the attributes of tools and outcome data comparing tools to clinical practice. We reported outcome data separately for those tools compared with an alternative method to systematically collect family history, considered by the investigators to be a more "optimal" approach than the tool being evaluated, and those compared with current "standard" primary care practice. In studies using "optimal" comparators, the comparator was information collected by a clinical genetic specialist or structured interviews administered by trained researchers; when current practice was the comparator, this information was collected in a way that was "standard" for the primary care setting (e.g., family history information recorded in patient charts).

\section{Role of the funding source}

The study sponsor (Agency for Healthcare Research and Quality) provided feedback on peer reviewers, draft and final versions of the related evidence report.

\section{RESULTS}

\section{Studies reviewed}

A total of 39 different family history tools, implemented in 40 unique studies, and reported in 45 publications passed full text criteria. Our initial focus was on identifying studies that described tools developed or used in a primary care clinical setting 16,17,19-25; however, after careful review, we noted that many studies described tools used in other settings that seemed potentially applicable to primary care (as defined previously). We sent email requests for copies of the tool to all authors of eligible studies who did not provide sufficient detail about the tool in the publication. Fifteen authors (of 16 publications ${ }^{26-41}$ ) did not respond in time for the publication of this review, and therefore, we were unable to determine whether the reported tools were applicable for use within primary care. Six tools from seven publications ${ }^{42-48}$ were assessed as inappropriate for primary care. Of the remaining 22 publications, four ${ }^{22-25}$ described the prototype and final versions of the same primary care tool, which we counted as a single tool, and two ${ }^{49,50}$ were companion publications. Thus, from 22 publications, 18 distinct tools were identified, eleven developed for primary care populations and a further seven developed for other settings but applicable to primary care (Fig. 1). Nine web-based clinical tools were also identified.

\section{Description of tool}

As indicated in Table 1, 14 tools ${ }^{16,17,19,21,51-60}$ were designed for completion by patients, with three studies reporting that these were completed in the PCPs office. ${ }^{16,17,54} \mathrm{~A}$ further four tools (eight publications $20,22-25,49,50,61$ ) were designed for use by health professionals. Four tools were described (seven publications ${ }^{16,19,20,22-25}$ ) which were implemented in family practice settings, and four tools 17,21,59,61 in internal medicine clinics. One tool ${ }^{54}$ was implemented in a screening mammography setting, and another four tools ${ }^{55-58}$ were designed for use in cancer centers or clinics. A further three ${ }^{51-53}$ were implemented in genetic clinics. One tool (two publications ${ }^{49,50}$ ) was web based and designed for use by any health professional, and the remaining tool ${ }^{60}$ was used in a large population-based research study.

\section{Cancer type identified and purpose of family history tool}

On reviewing details of the tool in publications and actual tool (when available), 14 tools, reported in 17 articles, ${ }^{17,19-25,49-55,57,59,60}$ were designed to collect data on family history of breast or breast/ ovarian cancer. Ten tools (12 publications $\left.{ }^{17,19,22,25,49-51,55,57,59-61}\right)$ specifically captured data on colorectal cancer and five tools (six publications $17,19,49-51,57)$ on prostate cancer. Nine tools (10 publications $\left.{ }^{19,20,22,25,51,52,55,57,60,61}\right)$ also captured data on one or more additional cancer types. For three, ${ }^{16,56,58}$ the tool seemed to invite information on any cancer type.

The output of five tools (nine publications $22-25,49,50,53,54,57$ ) was linked directly to some form of defined risk assessment (i.e., the family history data were converted directly into a risk categorization). Three of these tools were developed for primary care populations. ${ }^{22-25,49,50,54}$ 


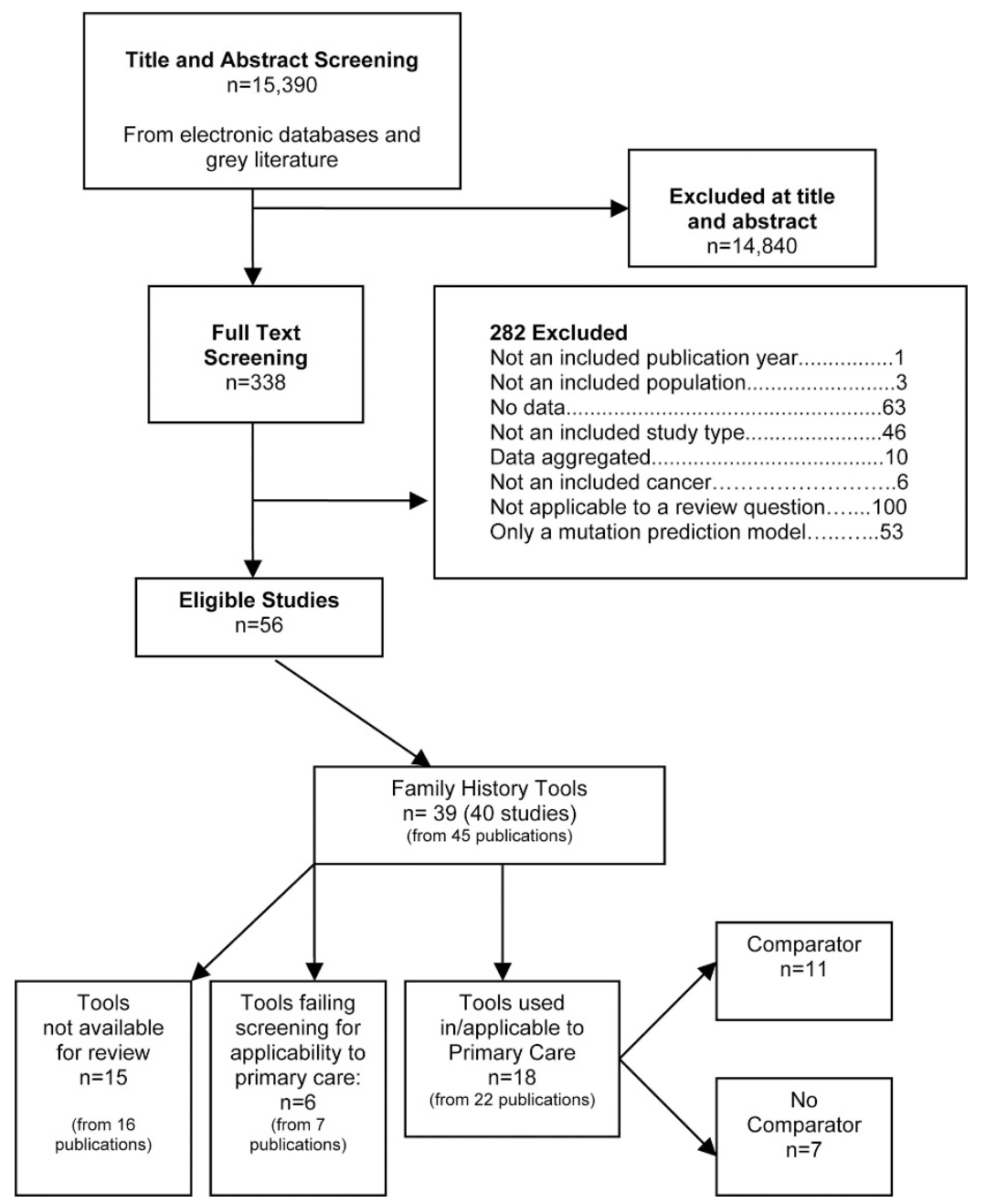

Fig. 1. Publications identified, selected, and excluded.

\section{Format}

Eleven tools ${ }^{16,17,19,21,52,54-56,58-60}$ were paper based, generally in some form of questionnaire or structured questions. Four tools (eight publications ${ }^{22-25,49,50,53,57}$ ) were presented in a format for use on a desktop or laptop computer, including web based and touch screen applications, and one on a personal digital assistant. ${ }^{61}$ Two of these electronic tools required the patient to initially complete a self-administered paper-based family history questionnaire before inputting data. ${ }^{22,25,53}$ One tool $^{51}$ was an automated telephone interview, and one was a structured interview schedule. ${ }^{20}$

\section{Content and complexity}

Sixteen tools ${ }^{16,19-25,51-61}$ reported in 19 publications were designed to capture data on all or selected 1DRs.

Thirteen tools (16 articles $\left.{ }^{16,20-25,51,53-57,59-61}\right)$ were designed to capture data on all or some second degree relatives (2DRs), and one ${ }^{16}$ on grandparents only. Six tools ${ }^{16,51,53-55,57}$ explicitly went beyond 2DRs, although not necessarily to capture all third degree relatives (3DR). For the remaining tools, the extent of family history enquiry was not explicitly described.

For all tools, except five, ${ }^{19,56,58,60,61}$ there were explicit instructions for users to capture data on relatives on both sides of the family. Seven tools $16,17,21,22,25,51,56,57$ were designed to capture ethnicity data. In most tools, age of diagnosis of affected relatives was reported, whereas five tools reported directing the user to enquire about unaffected relatives. ${ }^{16,51,56,58,60}$ The respondents were asked to score the certainty with which they recalled relatives' medical information in one of the tools developed for primary care. ${ }^{20}$ Nine tools reported collating respondents' past medical histories: this varied from general enquiry in three tools, ${ }^{16,17,59}$ with two tools focusing on specific cancers ${ }^{19,60}$ and five identifying personal risk factors. ${ }^{19,51,56,57}$ The complexity of the paper-based tools varied from a sequence of 6-10 closed questions, ${ }^{19,54}$ to a more detailed questionnaire varying in size from two ${ }^{55}$ to nine pages. ${ }^{56}$ In some cases, the questions were presented in tabular form, ${ }^{16,17,56,59}$ whereas two of the electronic tools presented the information as pedigrees..$^{22-25,49-51}$

\section{Other family history tools}

Eleven web-based tools were also identified during the gray literature search. Nine tools were actually available on the web, and these are listed with relevance scores in the full report. ${ }^{18}$ For all except one (JamesLink), ${ }^{57}$ which was included in the main review, no information was provided on their development or evaluation, which precluded their inclusion in the main review. 


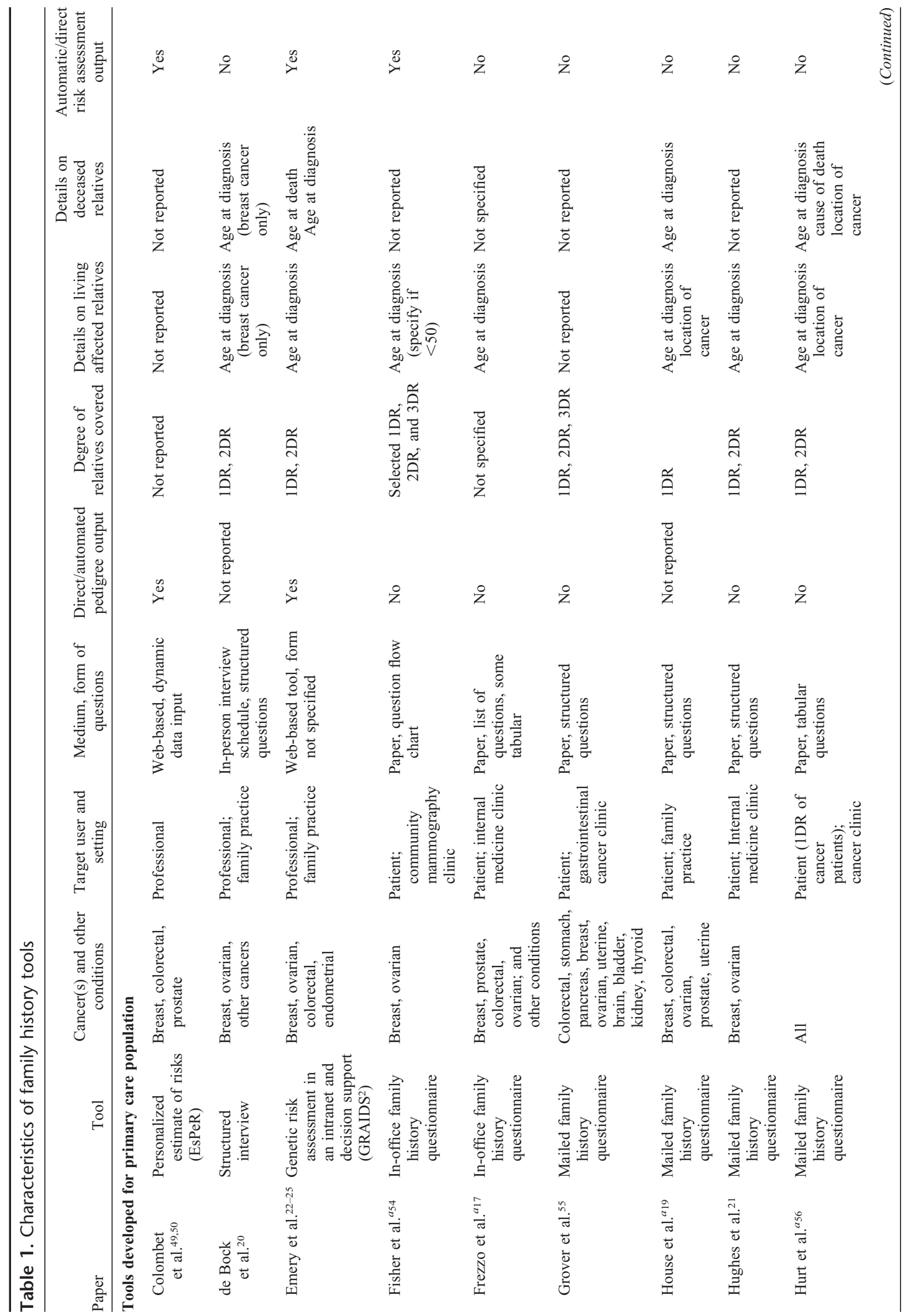




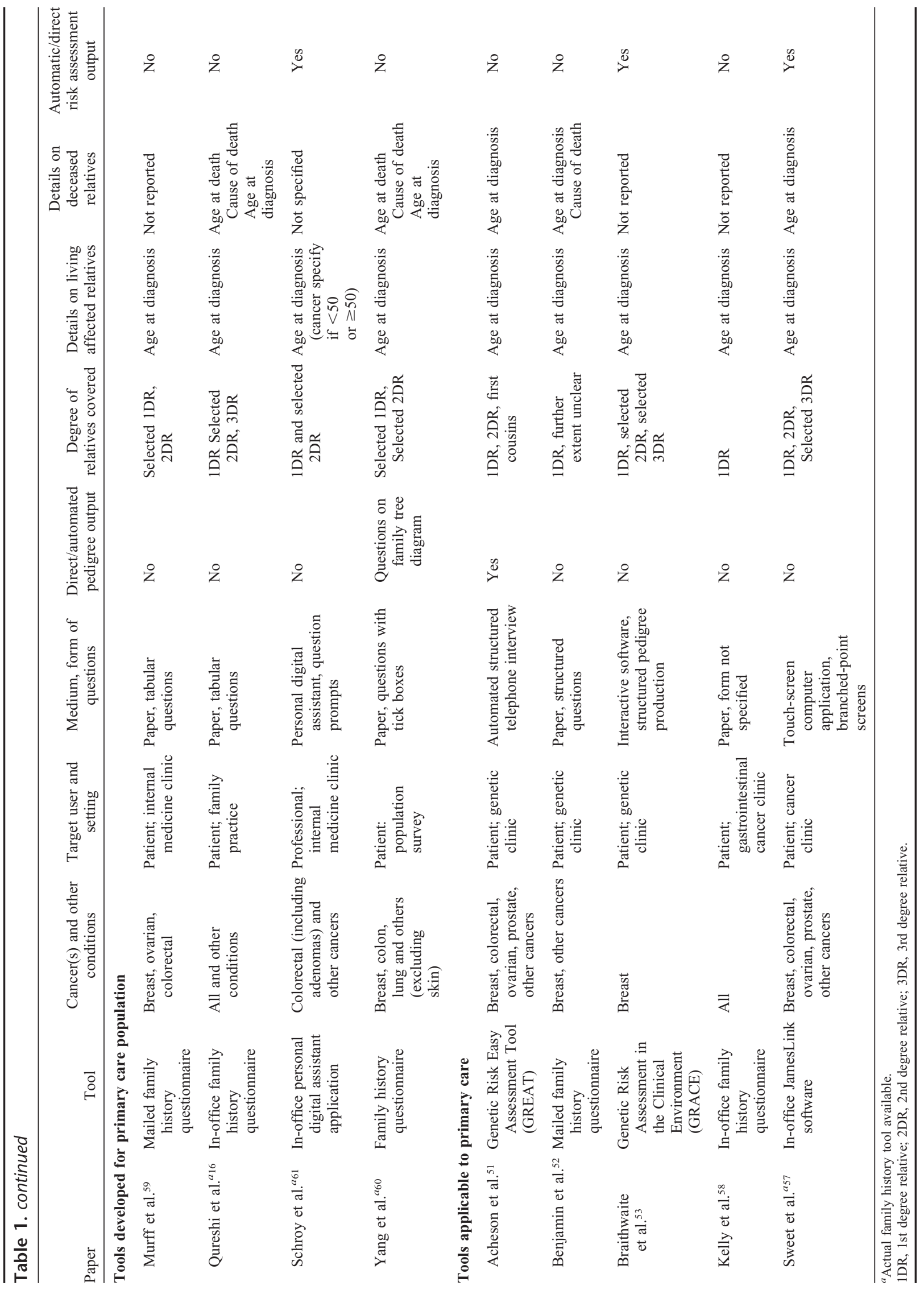


The highest scoring of these tools for applicability to primary care were the Family History Tool developed by the American Academy of Family Practice ${ }^{62}$ and the US Surgeon General's Family History Initiative. ${ }^{63}$

\section{Evaluating the family history collection tools}

The tools were evaluated using a range of study designs and settings (Table 2). The term "controlled" indicates study design where there are at least two arms, one of which is the tool in question and the other an alternative method of capturing family history data.

\section{Validity and reliability}

Six tools (nine publications) were described as having undergone a development or piloting phase. ${ }^{16,19,22-25,51,54,58}$ Five studies assessed acceptability and ease of completion of the tool. $22,25,51-53$ Qualitative techniques were also described to assess face validity in studies of four tools, including semistructured interviews with practitioners ${ }^{23,24}$ and patients, ${ }^{16}$ and focus groups with practitioners. ${ }^{16,49,50}$ Three studies reported the length of time needed to complete the tool, ranging from $8^{54}$ to around 30 minutes. ${ }^{51,53}$ One study, in a genetic clinic setting, ${ }^{51}$ reported test-retest reliability of $97 \%$ (401/413) for 1DRs, and $93 \%(620 / 667)$ for 2DRs, respectively, and 98\% (322/328) for cancers identified.

\section{Outcomes}

There were six family history tools (seven publications) without a comparator group or control arm ${ }^{19-21,49,50,56,60}$ and one tool in a controlled study but no comparator to capture family history data. ${ }^{53}$ These seven descriptive publications comprised two evaluating familial cancer risk assessment, ${ }^{56,60}$ two studies (three publications) only evaluating the content and face validity of the tools, ${ }^{49,50,53}$ and three publications ascertaining the prevalence of respective familial cancer histories. ${ }^{19-21}$ The performance of the 11 remaining tools was assessed in some way against a defined comparator. The tool was given first followed by the alternative "optimal" approach to systematic family history collection in three studies. ${ }^{16,52,54}$ The two crossover studies did not take place in primary care but determined that the order of completion was not associated with differences in outcomes. ${ }^{51,58}$ For studies where concealment was necessary in qualitative assessment of the tool, only two studies provided evidence that this had been performed. ${ }^{16,52}$ Full quality assessment of the studies is reported elsewhere. ${ }^{18}$

Evaluation against an "optimal" genetic interview was undertaken for five tools ${ }^{16,51,52,54,58}$ (Table 2). Two tools were developed for primary care: Fisher compared the primary care interview to a genetic interview, ${ }^{54}$ whereas Qureshi et al. ${ }^{16}$ compared a self-completed questionnaire to a structured genetic interview administered by a trained researcher. The three other tools, identified from other settings, compared the tool against clinical genetic interviews in two studies ${ }^{51,52}$ and compared against the identical interview schedule in the third study. ${ }^{58}$ The measures used to evaluate tools against these genetic or structured interviews included: percentage agreement, ${ }^{16,51,54}$ sensitivity, specificity, 16,52 Kendal's Correlation ${ }^{51}$ and Cohen's Kappa analysis. ${ }^{16,51}$ Most of these metrics assumed the alternative interview was the "gold standard" measure. The respective results are included in Table 2 . The percentage agreement was for specific attributes of family history, such as number of relatives diagnosed, ${ }^{16,51}$ type of cancers identified, ${ }^{51}$ and risk categorization. ${ }^{16,51,54}$ Considering the tools developed for primary care, the percentage agreement on risk stratification varied from $77 \%(93 / 121)^{16}$ to $100 \%(89 / 89) .{ }^{54}$ This was similar to the
$75 \%(90 / 120)$ agreement for a tool used in the genetic setting. ${ }^{51}$ Further, in Qureshi, ${ }^{16}$ Kappa ranged from 0.10 to 0.70 , this corresponding to more 2DRs identified in $51 \%$ of interviews $(62 / 121)$ and more $1 D R s$ in $15 \%$ of interviews (18/121). However, in Fisher, ${ }^{54}$ of 45 women classified at population risk by the tool, none were reassigned at higher risk by the genetics interview, and of 45 women classified at elevated risk, none were reclassified as population risk. Similarly, in the clinical genetic setting, the tool developed by Benjamin identified $92 \%{ }^{64}$ of the 84 identified at increased cancer risk by genetic interview. ${ }^{52}$

Evaluation against "current standard" practice was performed for six tools (seven publications $17,23,24,55,57,59,61$ ). Four tools were developed for primary care, $17,23,24,59,61$ and the other tools for use in cancer clinics. ${ }^{55,57}$ One of the latter tools has also been adopted for use by the public as a web-based tool (as discussed later). ${ }^{57}$ Current practice included the family history as recorded in patient charts, ${ }^{17,55,57,59,61}$ and accuracy or completeness of pedigrees derived from simulated patient histories drawn without access to a tool. ${ }^{23}$ The study findings are displayed in Table 2. In Frezzo et al., ${ }^{17}$ the outcome measure was the percentage agreement for identifying high-risk individuals between the patients' charts and the tool, reporting $78 \%$ better identification of significant family histories when the tool was used in an internal medicine clinic. This was compared with a $46 \%$ improvement in the cancer clinic setting. ${ }^{57}$ In Frezzo et al., ${ }^{17}$ of 32 patients at increased risk on tool, 18 were identified on patient charts; whereas in the cancer clinic setting, ${ }^{57}$ the corresponding numbers were of 101 patients on tool, 69 were identified on chart. The other cancer clinic study presented concordance rates ${ }^{55}$ and identified that a third of patient charts missed cancers in 1DRs captured by the tool. Emery et al. ${ }^{23}$ describe the development of a primary care family history/risk assessment tool with data reported from an evaluation of the prototype (RAGS), in which 36 family physicians used three different methods to draw pedigrees and assess the risk of simulated patients. Pedigrees produced using the RAGS tool were statistically significantly more likely to be accurate than those prepared by a genetics software package (Cyrillic) or by traditional pen and paper methods (median correct pedigrees, 5/6 for RAGS, 3.5/6 for Cyrillic, 2/6 for pen and paper).

\section{DISCUSSION}

This review explores the effectiveness of tools for collecting familial cancer history that could be used in a primary care setting. Ideally, patients are able to report accurate information on their family history, assisted by effective tools, and health care providers are able to use the information to make beneficial preventive and clinical management decisions. The review identified a number of family history collection tools developed for use in a primary care setting, most of which had not been evaluated against "optimal" or standard primary care practice.

Because of the limited number of studies, the evaluation of family history tools was extended to relevant tools in nonprimary care settings. Sixty-one percent of the tools $(11 / 18)$ were evaluated against current practice. There was reasonable agreement between the tools and measures of optimal or gold standard; however, there was limited consistency between studies on the standard chosen. A gold standard in clinical practice may be considered the pedigree-drawing interview performed by a genetic specialist, whereas in two of the identified studies, structured interviews administered by nonspecialists were used as surrogate approaches to the genetic interview. ${ }^{16,58}$ If we are 


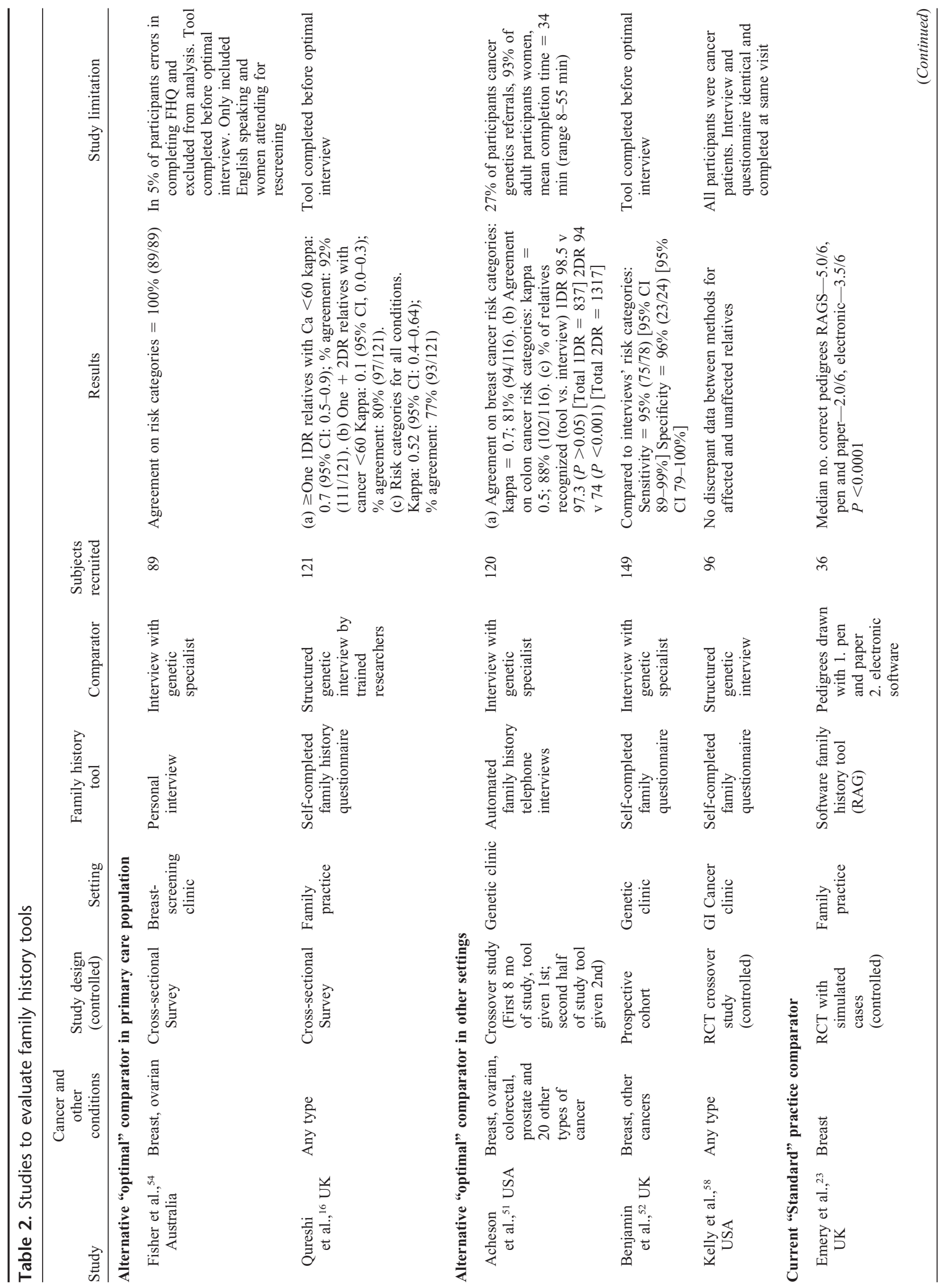




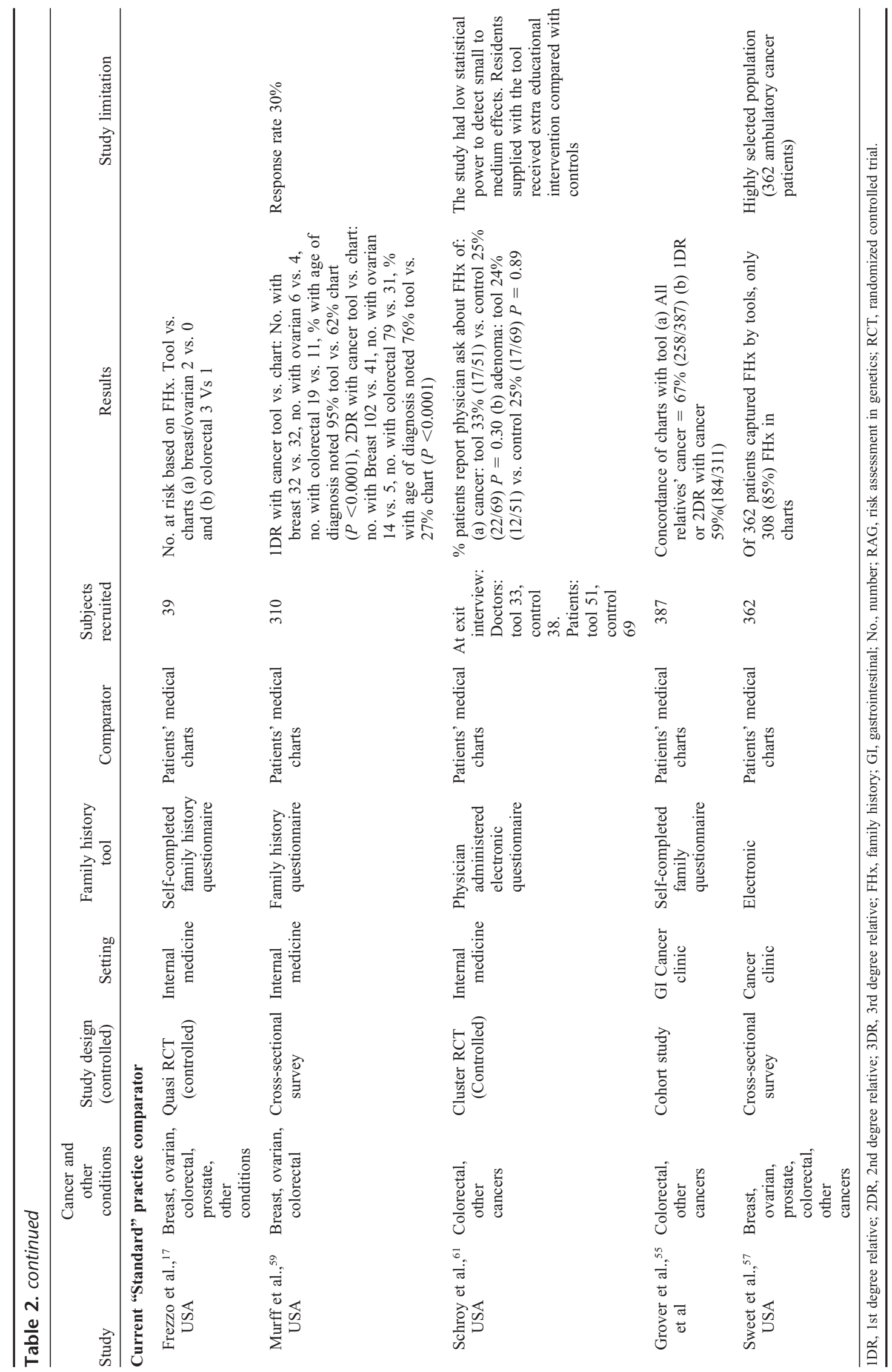


evaluating the PCPs ability to emulate the standard of the genetic specialist pedigree-drawing interview then we have to read the findings of these studies with caution. However, as discussed later, this may not always be the role of the primary care tool. When compared with current standard practice, primary care tools identified significantly more relatives with cancer. Although only limited to a few studies, comparing tools with family history in patients' charts, the improvement in recording of family history was greater for the primary care tool than those administered in specialist cancer settings. ${ }^{17,55,57}$ In some cases, this would lead to reassignment of risk category and altered prevention plans. The clinical significance and added benefit of this information still needs to be explored.

The tools identified in this review varied considerably, from those which took a comprehensive approach, emulating the geneticist's pedigree-drawing interview, to those which focused on identifying selected cancers in specific relatives. With the limited studies available, it was not possible to compare the utility of tools designed to collect family history on single cancers to those that identified several conditions. Many tools were designed to be used in the physician's office, in paper based or electronic format. It has been suggested that other formats, such as web based or mailed surveys, allow patients to (potentially) take "ownership" of their family history, offer them the opportunity to gather information from relatives, and may make for better use of PCP time. ${ }^{16,22,25,51,52,54,59}$ Several organizations have set up similar web-based tools for public use. ${ }^{57,65-69}$ It is unfortunate that in 10 of the 11 applicable web-based tools, no published information on their development or evaluation was available. Of these, the tools developed by the American Academy of Family Practice 62 and the US Surgeon General's Family History Initiative ${ }^{63}$ scored highest on assessment of applicability to primary care. These may potentially be the type of tools that will be used by those providers with electronic medical records.

The acceptability and ease of completion of family history tools were assessed in only a few studies. These aspects of the tools' content and face validity should be an integral part of evaluating any future primary care tools. Pedigrees are the standard clinical genetic approach to present family history information; however, tabular format and lists may also be appropriate in the primary care setting, particularly in settings using electronic medical records. ${ }^{16,57}$

Accurate risk assessment generally requires information on the side of the family (maternal, paternal, or both) to which relatives with cancer belong, and most tools identified this. Further, ethnicity (an indication of ancestry ${ }^{70}$ ) may be associated with increased risk of particular cancers, but few tools were designed to capture such data on ethnicity. We suggest that, in future tool development studies, it might not be necessary to replicate the extent or type of data captured in a clinical genetics setting. Further, it would be useful to distinguish between two different purposes for tools - assembly and updating of "complete" family history information in a generic approach, and ascertainment of targeted information for specific disease risk assessment. For the latter, an "adequate" family history requires not only identifying relatives with and without the cancer but also the relationship of the affected relative, the age of onset of cancer in those affected, and identification of several cancer types beyond the "target" cancer in question (e.g., family history of endometrial and kidney cancer is relevant in considering risk for hereditary nonpolyposis colorectal cancer). Further, it may be logical to evaluate the performance of such tools as part of a disease-specific risk assessment tool, rather than as a stand-alone tool.
Considering the former generic use of these tools (where a specific condition is not being targeted), approaches to the tool's rational development would benefit from agreement on the "minimum family history dataset," bearing in mind that the goal in this primary care setting is usually to identify an index of suspicion for genetic predisposition, leading to more targeted enquiries if a possible familial cancer risk is identified. Based on the few studies identified in the review, it is not possible to recommend any specific family history tool. Further, choice will depend on purpose of the tool, patients' and providers' computer access, and personal preference. However, at the very least, family history information should include both sides of the family, all 1DRs, ethnicity and age of diagnosis of affected relatives. Table 3 lists some of the elements, which could be considered for inclusion in a more comprehensive dataset. It is presented to foster consensus about the extent of family history enquiry necessary for different clinical purposes and circumstances, taking into account the likelihood of accuracy of selfreported information for different relatives. ${ }^{71}$ For instance, although some authors have suggested that a minimum family history should cover three generations, $3,71,72$ the reliability of information beyond 1DRs and grandparents is not well evaluated and would suggest decreased accuracy. ${ }^{35,46,47,73,74}$

Further, family histories are not static ${ }^{16,54}$; however, practical issues of updating family history have not been explored. On the one hand, PCPs may be able to assemble a patient's family history information over time, but on the other, necessary updates consume time and resources. Acheson et al. ${ }^{2}$ has reported that most family histories were completed on the first visit. It would be worth considering formally whether a staged approach over several visits leads to more accurate or extensive information, and clarifying the optimum interval for updates. Barriers to the use of tools in practice also need considering. These include lack of time, ${ }^{75}$ lack of PCPs' confidence in their knowledge and skills in genetics, ${ }^{76}$ and reimbursement policies. ${ }^{3}$

It seems logical that family history tools are likely to produce most benefit if they are accompanied by risk assessment and management plans for patients at increased risk of familial cancer; otherwise, family history collection by PCPs and/or consumers may be wasteful of time, energy, health care resources, and may even be harmful. Although some guidelines ${ }^{77}$ recommend that family history information should only be collected in response to patient enquiry about familial breast cancer risk or if the provider suspects increased cancer risk, others argue that family history collection is an integral part of good clinical practice in primary care and that failure to do so should be considered negligence. ${ }^{57,58,71}$

In addition to using several web-based search engines, our search of relevant gray literature was constrained to sites specified by the investigators, our technical expert panel, and peer reviewers. Further, the studies reviewed in this report were limited to those published in English. However, our peer-review process allowed content experts in this area to identify any additional studies (both published and unpublished) of relevance for this systematic review thereby minimizing the likelihood of publication bias. Language bias also limited the ability to interpret non-English tools; however, this had a minimal impact on the studies described and evaluated. Our criteria for defining applicability to primary care were developed within our investigative team and have not been subject to external scrutiny. Further, the interpretation of the studies retrieved is limited because no study confirmed the family history collected by the tools with the relatives' actual medical histories.

Future tool development needs to integrate with other clinical and office systems, and consider contextual factors which might 
Table 3. Potential Items for Inclusion in a Family History Dataset for a Generic Family History Tool

(a) Relatives on whom data may be captured

\begin{tabular}{|c|c|}
\hline \multirow{3}{*}{$\begin{array}{l}\text { Degree of } \\
\quad \text { relatedness }\end{array}$} & Relationship \\
\hline & Informant $^{a}$ \\
\hline & Spouse/partner ${ }^{b}$ \\
\hline \multirow{3}{*}{$\begin{array}{l}\text { First degree blood } \\
\text { relatives }\end{array}$} & Mother, father \\
\hline & Brothers, sisters \\
\hline & Sons, daughters \\
\hline \multirow{4}{*}{$\begin{array}{l}\text { Second degree blood } \\
\text { relatives }\end{array}$} & Grandparents (both sides) \\
\hline & Aunts and uncles (both sides) \\
\hline & Half-brothers and half-sisters \\
\hline & Grandchildren \\
\hline \multirow{2}{*}{$\begin{array}{l}\text { Third degree blood } \\
\text { relatives }\end{array}$} & Cousins (both sides) \\
\hline & Nephews and nieces (both sides) \\
\hline
\end{tabular}

(b) Items of information that may be captured

Individual Item

Informant/patient Age or date of birth

History of cancer, for each:

Age at diagnosis

Specific information (e.g., bilaterality)

History of other relevant medical conditions (depending on cancer)

Results of relevant investigations, including genetic tests

Ethnicity or ancestry:

Self-identified ethnic group

Ethnic group of grandparents

Relatives

History of cancer, for each:

Age at diagnosis

Specific information (e.g., bilaterality)

Source/certainty of information

History of other relevant medical conditions (depending on cancer)

History of relevant investigations, including genetic tests

Living relatives Current age or date of birth

Deceased relatives Age at death

\section{Source of information}

Certainty of information

Cause of death

Source of information

Certainty of information

${ }^{a}$ Personal medical history important in risk assessment.

${ }^{b}$ May be relevant in respect of environmental and lifestyle/behavioral aspects of risk assessment. moderate their effect, and their marginal benefit in practice. The latter includes research to identify the specific strategies (e.g., sending tools home with patients) and tool features, which promote the most accurate reporting of family history information. Further, studies to evaluate tools should consider the benefits, costs, and harms of using patient-completed tools for systematic family history collection, as a substitute for, or complement to, tools designed for completion by health care providers. In addition to assessing technical outcomes such as accuracy and completeness of data captured, evaluations should consider outcomes, which relate to utility in routine practice, such as patient "empowerment." In designing these studies, researchers should also adopt appropriate comparators (generally current practice) and ensure that tools are optimized (in terms of, for example, face and content validity) before evaluation.

In summary, family history is a fundamental element of health information, and the ability to take an adequate and accurate cancer family history should be recognized as a core skill for all PCPs, irrespective of the availability of tools. Very few tools have been developed for, and evaluated in, primary care settings. Further, few tools have been compared with either gold standard (genetic interview) or current primary care "standard practice" (family history as recorded in charts). Although the evidence is very limited, and depends on extrapolation from studies of tools in settings other than primary care, it suggests that systematic tools may add significant family health information compared with current primary care practice.

\section{ACKNOWLEDGMENTS}

This systematic review was funded by the Agency for Healthcare Research and Quality, United States Department of Health and Human Services (contract no. 290-02-0020). Nadeem Qureshi had full access to all of the data in the study and takes final responsibility for submission of the paper and the accuracy of the data analysis. Nadeem Qureshi leads a UK NHS R\&D program on Clinical Genetics in Primary Care, and Parminder Raina holds a Canadian Institute of Health Research Investigator award, and an Ontario Premier's Research Excellence award.

\section{REFERENCES}

1. Yoon PW, Scheuner MT, Peterson-Oehlke KL, Gwinn M, Faucett A, Khoury MJ. Can family history be used as a tool for public health and preventive medicine? Genet Med 2002;4:304-310.

2. Acheson LS, Wiesner GL, Zyzanski SJ, Goodwin MA, Stange KC. Family history-taking in community family practice: implications for genetic screening. Genet Med 2000;2:180-185.

3. Rich EC, Burke W, Heaton CJ, et al. Reconsidering the family history in primary care. J Gen Intern Med 2004;19:273-280.

4. Scheuner MT, Gordon OK. Genetic risk assessment for common diseases Emery and Rimoin's Principal and Practice of Medical Genetics, 4th ed. London: Churchill-Livingstone, 2002:654-674.

5. Johns LE, Houlston RS. A systematic review and meta-analysis of familial colorectal cancer risk. Am J Gastroenterol 2001;96:2992-3003.

6. Sandhu MS, Luben R, Khaw KT. Prevalence and family history of colorectal cancer: implications for screening. J Med Screen 2001;8:69-72.

7. Claus EB, Risch NJ, Thompson WD. Age at onset as an indicator of familial risk of breast cancer. Am J Epidemiol 1990;131:961-972.

8. Mettlin C, Croghan I, Natarajan N, Lane W. The association of age and familial risk in a case-control study of breast cancer. Am J Epidemiol 1990; 131:973-983.

9. Offit K, Brown K. Quantitating familial cancer risk: a resource for clinical oncologists. J Clin Oncol 1994;12:1724-1736.

10. Struewing JP, Hartge P, Wacholder S, et al. The risk of cancer associated with specific mutations of BRCA1 and BRCA2 among Ashkenazi Jews. $N$ Engl J Med 1997;336:1401-1408.

11. Moslehi R, Chu W, Karlan B, et al. BRCA1 and BRCA2 mutation analysis of 208 Ashkenazi Jewish women with ovarian cancer. Am J Hum Genet 2000; $66: 1259-1272$. 
12. Satagopan JM, Offit K, Foulkes W, et al. The lifetime risks of breast cancer in Ashkenazi Jewish carriers of BRCA1 and BRCA2 mutations. Cancer Epidemiol Biomarkers Prev 2001;10:467-473.

13. Warner E, Foulkes W, Goodwin P, et al. Prevalence and penetrance of BRCA1 and BRCA2 gene mutations in unselected Ashkenazi Jewish women with breast cancer. J Natl Cancer Inst 1999;91:1241-1247.

14. Lucassen A, Watson E, Harcourt J, Rose P, O'Grady J. Guidelines for referral to a regional genetics service: GPs respond by referring more appropriate cases. Fam Pract 2001;18:135-140.

15. Rose PW, Murphy M, Munafo M, Chapman C, Mortensen N, Lucassen A. Improving the ascertainment of families at high risk of colorectal cancer: a prospective GP register study. Br J Gen Pract 2004;54:267-271.

16. Qureshi N, Bethea J, Modell B, et al. Collecting genetic information in primary care: evaluating a new family history tool. Fam Pract 2005;22:663669.

17. Frezzo TM, Rubinstein WS, Dunham D, Ormond KE. The genetic family history as a risk assessment tool in internal medicine. Genet Med 2003;5: 84-91.

18. Qureshi N, Wilson B, Santaguida P, et al. Collection and use of cancer family history in primary care. Evid Rep Technol Assess 2007;157:1-84.

19. House W, Sharp D, Sheridan E. Identifying and screening patients at high risk of colorectal cancer in general practice. J Med Screen 1999;6:205-208.

20. de Bock GH, Perk DC, Oosterwijk JC, Hageman GC, Kievit J, Springer MP. Women worried about their familial breast cancer risk-a study on genetic advice in general practice. Fam Pract 1997;14:40-43.

21. Hughes KS, Roche C, Campbell CT, et al. Prevalence of family history of breast and ovarian cancer in a single primary care practice using a selfadministered questionnaire. Breast J 2003;9:19-25.

22. Emery J The GRAIDS trial: the development and evaluation of computer decision support for cancer genetic risk assessment in primary care. Ann Hum Biol 2005;32:218-227.

23. Emery J, Walton R, Murphy M, et al. Computer support for interpreting family histories of breast and ovarian cancer in primary care: comparative study with simulated cases. BMJ 2000;321:28-32.

24. Emery J, Walton R, Coulson A, Glasspool D, Ziebland S, Fox J. Computer support for recording and interpreting family histories of breast and ovarian cancer in primary care (RAGs): qualitative evaluation with simulated patients. BMJ 1999;319:32-36.

25. Emery J, Morris H, Goodchild R, et al. The GRAIDS Trial: a cluster randomised controlled trial of computer decision support for the management of familial cancer risk in primary care. Br J Cancer 2007;97:486-493.

26. Andrieu N, Launoy G, Guillois R, Ory-Paoletti C, Gignoux M. Estimation of the familial relative risk of cancer by site from a French population based family study on colorectal cancer (CCREF study). Gut 2004;53:1322-1328.

27. Bruner DW, Baffoe-Bonnie A, Miller S, et al. Prostate cancer risk assessment program. A model for the early detection of prostate cancer. Oncology (Huntington) 1999;13:325-334.

28. Chalmers KI, Luker KA, Leinster SJ, Ellis I, Booth K. Information and support needs of women with primary relatives with breast cancer: development of the Information and Support Needs Questionnaire. J Adv Nurs 2001;35:497-507.

29. De Jong AE, Vasen HF. The frequency of a positive family history for colorectal cancer: a population-based study in the Netherlands. Netherlands J Med 2006;64:367-370.

30. Eerola H, Blomqvist C, Pukkala E, Pyrhonen S, Nevanlinna H. Familial breast cancer in southern Finland: how prevalent are breast cancer families and can we trust the family history reported by patients? Eur J Cancer 2000;36:11431148

31. Fletcher RH, Lobb R, Bauer MR, et al. Screening patients with a family history of colorectal cancer. J Gen Intern Med 2007;22:508-513.

32. Green RC, Green JS, Buehler SK, et al. Very high incidence of familial colorectal cancer in Newfoundland: a comparison with Ontario and 13 other population-based studies. Fam Cancer 2007;6:53-62.

33. King TM, Tong L, Pack RJ, Spencer C, Amos CI. Accuracy of family history of cancer as reported by men with prostate cancer. Urology 2002;59:546-550.

34. Skinner CS, Rawl SM, Moser BK, et al. Impact of the Cancer Risk Intake System on patient-clinician discussions of tamoxifen, genetic counseling, and colonoscopy. J Gen Intern Med 2005;20:360-365.

35. Theis B, Boyd N, Lockwood G, Tritchler D. Accuracy of family cancer history in breast cancer patients. Eur J Cancer Prev 1994;3:321-327.

36. Tischkowitz M, Wheeler D, France E, et al. A comparison of methods currently used in clinical practice to estimate familial breast cancer risks. Ann Oncol 2000;11:451-454.

37. Weinrich SP, Faison-Smith L, Hudson-Priest J, Royal C, Powell I. Stability of self-reported family history of prostate cancer among African American men. J Nurs Meas 2002;10:39-46.

38. Breuer B, Kash KM, Rosenthal G, Diemer K, Osborne MP, Miller DG. Reporting bilaterality status in first-degree relatives with breast cancer: a validity study. Genet Epidemiol 1993;10:245-256.
39. Katballe N, Juul S, Christensen M, Orntoft TF, Wikman FP, Laurberg S Patient accuracy of reporting on hereditary non-polyposis colorectal cancerrelated malignancy in family members. Br J Surg 2001;88:1228-1233.

40. Parent M, Ghadirian P, Lacroix A, Perret C. The reliability of recollections of family history: implications for the medical provider. J Cancer Educ 1997; 12:114-120.

41. Parent ME, Ghadirian P, Lacroix A, Perret C. Accuracy of reports of familial breast cancer in a case-control series. Epidemiology 1995;6:184-186.

42. Anton-Culver H, Kurosaki T, Taylor TH, Gildea M, Brunner D, Bringman D. Validation of family history of breast cancer and identification of the BRCA1 and other syndromes using a population-based cancer registry. Genet Epidemiol 1996;13:193-205.

43. Hlavaty T, Lukac L, Huorka M, Bezayova T, Duris I. Positive family history promotes participation in colorectal cancer screening. Bratisl Lek Listy 2005; 106:318-323

44. Kerber RA, Slattery ML. Comparison of self-reported and database-linked family history of cancer data in a case-control study. Am J Epidemiol 1997; 146:244-248

45. Quillin JM, Ramakrishnan V, Borzelleca J, Bodurtha J, Bowen D, Baer WD. Paternal relatives and family history of breast cancer. Am J Prev Med 2006;31:265-268.

46. Sijmons RH, Boonstra AE, Reefhuis J, et al. Accuracy of family history of cancer: clinical genetic implications. Eur J Hum Genet 2000;8:181-186.

47. Ziogas A, Anton-Culver H. Validation of family history data in cancer family registries. Am J Prev Med 2003;24:190-198.

48. Aitken J, Bain C, Ward M, Siskind V, MacLennan R. How accurate is self-reported family history of colorectal cancer? Am J Epidemiol 1995;141: $863-871$.

49. Colombet I, Dart T, Leneveut L, Zunino S, Menard J, Chatellier G. Combining risks estimations and clinical practice guidelines in a computer decision aid: a pilot study of the EsPeR system. Stud Health Technol Inform 2003;95:525-530.

50. Colombet I, Dart T, Leneveut L, Zunino S, Menard J, Chatellier G. A computer decision aid for medical prevention: a pilot qualitative study of the personalized estimate of risks (EsPeR) system. BMC Med Inform Decis Mak 2003;3:13.

51. Acheson LS, Zyzanski SJ, Stange KC, Deptowicz A, Wiesner GL. Validation of a self-administered, computerized tool for collecting and displaying the family history of cancer. J Clin Oncol 2006;24:5395-5402.

52. Benjamin C, Booth K, Ellis I. A prospective comparison study of different methods of gathering self-reported family history information for breast cancer risk assessment. J Genet Couns 2003;12:151-170.

53. Braithwaite D, Sutton S, Mackay J, Stein J, Emery J. Development of a risk assessment tool for women with a family history of breast cancer. Cancer Detect Prev 2005;29:433-439.

54. Fisher TJ, Kirk J, Hopper JL, Godding R, Burgemeister FC. A simple tool for identifying unaffected women at a moderately increased or potentially high risk of breast cancer based on their family history. Breast 2003;12:120127.

55. Grover S, Stoffel EM, Bussone L, Tschoegl E, Syngal S. Physician assessment of family cancer history and referral for genetic evaluation in colorectal cancer patients. Clin Gastroenterol Hepatol 2004;2:813-819.

56. Hurt GJ, McQuellon RP, Michielutte R, Conrad DM, Carter S, Anderson H. Risk assessment of first-degree relatives of women with breast cancer: a feasibility study. Oncol Nurs Forum 2001;28:1097-1104.

57. Sweet KM, Bradley TL, Westman JA. Identification and referral of families at high risk for cancer susceptibility. J Clin Oncol 2002;20:528-537.

58. Kelly KM, Shedlosky-Shoemaker R, Porter K, Remy A, DeSimone P, Andrykowski MA. Cancer family history reporting: impact of method and psychosocial factors. J Genet Counsel 2007;16:373-382.

59. Murff HJ, Greevy RA, Syngal S. The comprehensiveness of family cancer history assessments in primary care. Commun Genet 2007;10:174180.

60. Yang Q, Khoury MJ, Rodriguez C, Calle EE, Tatham LM, Flanders WD. Family history score as a predictor of breast cancer mortality: prospective data from the cancer prevention study II, United States, 1982-1991. Am J Epidemiol 1998; 147:652-659.

61. Schroy PC, Glick JT, Geller AC, Jackson A, Heeren T, Prout M. A novel educational strategy to enhance internal medicine residents' familial colorectal cancer knowledge and risk assessment skills. Am J Gastroenterol 2005; 100:677-684.

62. American Academy of Family Practice, 2007. Family history tool. Available at: http://www.genetests.org//servlet/access?id $=8888892 \& \mathrm{key}=$

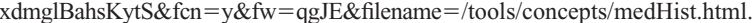
Accessed May 1, 2009

63. U.S. Surgeon General. My family health portrait. Rockville, MD: U.S. Department of Health \& Human Services, Office of the Surgeon General, 2007. 
64. McIntosh A., Shaw C, Evans G, et al. Clinical guidelines and evidence review for the classification and care of women at risk of familial breast cancer. National Institute for Clinical Excellence Clinical Guideline 14. London: National Collaborating Centre for Primary Care/University of Sheffield, 2004.

65. Guttmacher AE, Collins FS, Carmona RH. The family history-more important than ever. $N$ Engl J Med 2004;351:2333-2336.

66. Carmona RH, Wattendorf DJ. Personalizing prevention: the U.S. Surgeon General's Family History Initiative. Am Fam Phys 2005;71:36, 39.

67. Benkendorf J, Bodurtha J, Schreiber A. Virginia is for family history lovers. The genetic family history in practice 1 [Spring 2005], vol. 1. Lutherville, MD: National Coalition for Health Professional Education in Genetics (NCHPEG), 2005:8

68. Norwich Union, 2004. Norwich Union Health Tree. Available at: http:// www.norwichunion.com/healthtree/index.htm.

69. American Medical Association. AMA Adult Family History Form. Chicago, IL: American Medical Association, 2004.
70. Qureshi N, Kai J. Genomic medicine for underserved minority populations in family medicine. Am Fam Phys 2005;72:386-387.

71. Murff HJ, Spigel DR, Syngal S. Does this patient have a family history of cancer? An evidence-based analysis of the accuracy of family cancer history. JAMA 2004;292:1480-1489.

72. Bennett RL. The practical guide to the genetic family history. New York: Wiley, 1999.

73. Gaff CL, Aragona C, MacInnis RJ, et al. Accuracy and completeness in reporting family history of prostate cancer by unaffected men. Urology 2004;63:1111-1116.

74. Mitchell RJ, Brewster D, Campbell H, et al. Accuracy of reporting of family history of colorectal cancer. Gut 2004;53:291-295.

75. Summerton N, Garrood PV. The family history in family practice: a questionnaire study. Fam Pract 1997;14:285-288.

76. Watson EK, Shickle D, Qureshi N, Emery J, Austoker J The 'new genetics' and primary care: GPs' views on their role and their educational needs. Fam Pract 1999;16:420-425. 